\title{
Experimental Investigation of Size Effects in Colliding Droplets
}

\author{
Lars Pasternak ${ }^{* 1}$, Martin Sommerfeld ${ }^{2}$ \\ ${ }^{1}$ Otto-von-Guericke-Universität Magdeburg, Institut für Verfahrenstechnik, Germany \\ ${ }^{2}$ Otto-von-Guericke-Universität Magdeburg, Institut für Verfahrenstechnik, Germany \\ *Corresponding author: lars.pasternak@ovgu.de
}

\begin{abstract}
The present work focuses on the size effect of binary collisions of PVP droplets (viscosity $5.5 \mathrm{mPas}$ ). The aim was the identification of the boundaries between bouncing, coalescence, stretching and reflexive separation.

These boundaries are necessary for numerical simulations of droplet collisions in spray drying processes. Therefore, droplet chains were generated by droplet generators with oscillating membrane and directed towards each other at different angles for producing binary collisions. In the experiments two droplet properties (i.e. droplet size and size ratio) were varied. Two synchronised high-speed cameras were used to observe the collision process and outcome perpendicularly and parallelly to the collision plane. The variation of the impact parameter $B$ was performed by a frequency offset for one droplet generator. The relative velocity (i. e. 0.5 to $4.7 \mathrm{~m} / \mathrm{s}$ ) was set by changing the collision angle.
\end{abstract}

\section{Keywords}

droplet collision, droplet size ratio, modelling separation

\section{Introduction}

Spray drying processes constitute a very complex multiphase system due to the different elementary processes involved, i.e. atomization, drying, collision of droplets and formation of agglomerates. After atomization, droplet collisions with the different possible collision outcomes result in a modification on the product particle size distribution. Typical results of a collision of two droplets may be bouncing, coalescence and separation and were plotted in the well-known collision maps (see Figure 1). In these maps the non-dimensional impact parameter B (see Eq. 1) is plotted against collision Weber number (see Eq. 2). However, the collision map depends on droplet parameters, i.e. liquid and size properties of the droplets. For Euler/Lagrange calculation of sprays it is necessary to know the pattern of the collision map without running experiments.

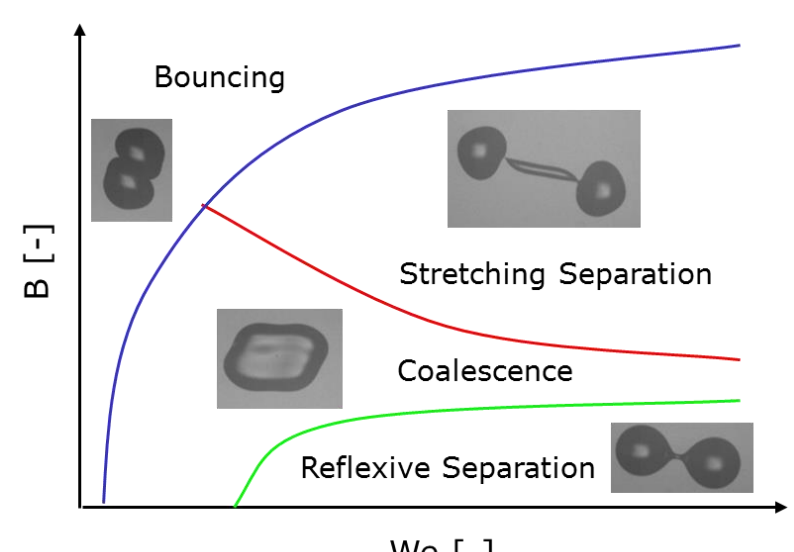

We $[-]$

Figure 1. Typical droplet collision map, with samples of collsion outcomes

During the last decades a number of models were developed to predict the collision outcome. The boundary between bouncing and coalescence/stretching separation can be identified by means of the experimental data based model by Estrade et al. [1]. The models by Ashgriz and Poo [2], for water droplets, are determining the lower boundaries for stretching separation and coalescence. The momentum theory based model by Jiang et al. [3] observes the viscosity effect for the lower boundary of the stretching separation. Based on the Jiang [3] model, Gotaas et al. [4] used experimental data from glycols to specify values of $C_{a}$ and $C_{b}$. This model was improved by 
Sommerfeld [5] by finding a dependency between $C_{a}$ and the Ohnesorge number (see Eq. 3) and setting the other involved constant $C_{b}=1$. The data from the experiments by Kuschel and Sommerfeld [6] were obtained only from $\sim 380 \mu \mathrm{m}$ mono-sized droplets. To validate the 3rd order polynomial fit of $\mathrm{C}_{\mathrm{a}}$ and Ohnesorge number, it was necessary to run experiments with variation of the droplet size properties. Furthermore, this study is devoted to describe the triple point, where all regimes are in coincidence, as well as the reflexive separation.

\section{Material and methods}

The experiments were carried out using two oscillating membrane droplet generators (producer: Encap BioSystems, model: IE-0010H-P). The Polyvinylpyrrolidone K17 solution (see Table 1) was supplied by a pressure vessel (see Figure 2). Droplet chains were created by means of excitation of the liquid inside the droplet generator and pressed throw 200, 300 and $400 \mu \mathrm{m}$ nozzles. The excitation frequency for the break-up was nozzle size dependant and in the range between 1800 and $4690 \mathrm{~Hz}$. The amplitude of the excitation signal was set by an amplifier (Thomann TA1050). A rotation stage was mounted on a three axis translation stage system in order to control the position of each nozzle. In combination with one of the two synchronised high-speed cameras (perpendicular to the collision plane) and the translation stage, off centre collisions could be avoid. The relative velocity (i. e. 0.5 to $4.7 \mathrm{~m} / \mathrm{s}$ ) was set by changing the droplet chain angle by the use of the rotation stages of each droplet generator.

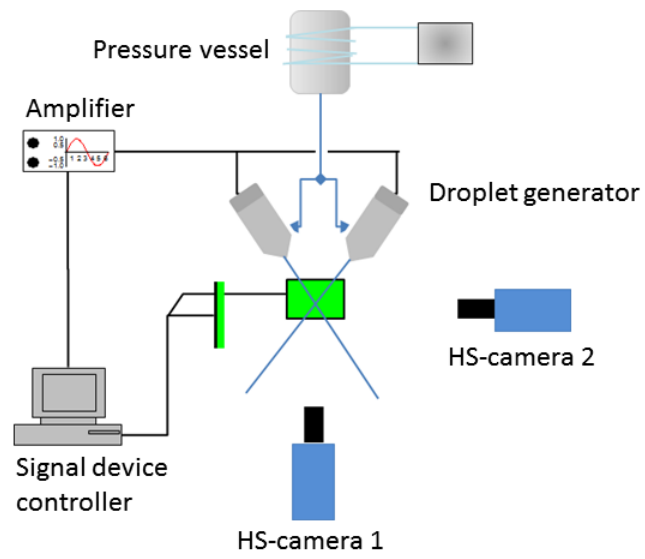

Figure 2. Sketch of the experimental setup [6]

Table 1. Liquid properties the PVP - Water solution at $22^{\circ} \mathrm{C}$

\begin{tabular}{c|cccc}
\hline \hline Specie & $\begin{array}{c}\text { Mass } \\
\text { fraction [\%] }\end{array}$ & $\begin{array}{c}\mathrm{M} \\
{[\mathrm{mPas}]}\end{array}$ & $\begin{array}{c}\sigma \\
{[\mathrm{mN} / \mathrm{m}]}\end{array}$ & $\begin{array}{c}\rho \\
{\left[\mathrm{kg} / \mathrm{m}^{3}\right]}\end{array}$ \\
\hline \hline PVP (BASF) & 20 & 5.5 & 62.2 & 1043.2
\end{tabular}

The illumination of the collision process was done by two $8 \times 10 \mathrm{~cm}^{2}$ backlight LED arrays. The observation of the droplet collision event was based on two Photron SA4 high speed cameras operating up to 10,000 frames per second. The synchronisation of the cameras was controlled by a signal generator. The cameras were equipped

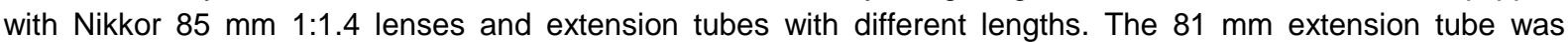
mounted on both cameras. For Case 1 (see Table 2) the tube on camera 1 was extended to $110 \mathrm{~mm}$. In the combination of lens and extension tube the resolution was 13.2 (only camera 1 in Case 1 ) and $17.2 \mu \mathrm{m} /$ pixel.

Table 2. Nozzle configuration for PVP droplets (see Table 1)

\begin{tabular}{c|c|ccccccc}
\hline \hline & $\begin{array}{c}\text { Nozzle } \\
\text { Combination } \\
{[\mu \mathrm{m}]}\end{array}$ & $\begin{array}{c}\text { Size } \\
\text { drop 1 } \\
{[\mu \mathrm{m}]}\end{array}$ & $\begin{array}{c}\text { Size } \\
\text { drop 2 } \\
{[\mu \mathrm{m}]}\end{array}$ & $\begin{array}{c}\text { Size } \\
\text { ratio } \\
\Delta[-]\end{array}$ & $\begin{array}{c}\text { Oh } \\
\text { drop 1 [-] }\end{array}$ & $\begin{array}{c}\text { Oh } \\
\text { drop 2 [-] }\end{array}$ & $\begin{array}{c}\text { max. Re } \\
\text { drop 1 [-] }\end{array}$ & $\begin{array}{c}\text { max. Re } \\
\text { drop 1[-] }\end{array}$ \\
\hline Case 1 & $200-200$ & 349 & 353 & 0.99 & 0.0364 & 0.0362 & 256 & 260 \\
Case 2 & $300-300$ & 560 & 557 & 0.99 & 0.0286 & 0.0289 & 502 & 490 \\
Case 3 & $300-400$ & 580 & 679 & 0.85 & 0.0282 & 0.0261 & 320 & 378 \\
Case 4 & $200-300$ & 411 & 563 & 0.73 & 0.0335 & 0.0286 & 234 & 310 \\
Case 5 & $200-400$ & 440 & 673 & 0.65 & 0.0324 & 0.0262 & 277 & 391
\end{tabular}


Due to the droplet velocity up to $4 \mathrm{~m} / \mathrm{s}$, the high resolution and the camera frame rate, the droplet displacement between two images was up to $400 \mu \mathrm{m}$. To avoid issues with the particle tracking it was necessary to develop a new approach for the detection of the droplet position. In the first image of both collision partners an eccentric box (see Figure 3 left, middle) is set around both droplets. The eccentric shape was necessary as a result of the different droplet velocities of both chains. This box ensures that only droplets inside are detected and only two collision partners are existing. In the next image the box is shifted by a default offset, by determining the droplet pixel velocities and droplet sizes the real offset is calculated for the next image. The positions and velocities from the droplets are based on their centre of mass. In the following images the offset is always recalculated, the collision possibility is verified and the size and shape from the box readjusted. The droplet detection ends in the last image before contact. Inside the box the droplets are identified by Laplacian of Gaussian operator, which limited the accuracy of the droplet position and size results. The expected error by the edge detection is less than one pixel. Due to the fluctuation of the instantaneous velocities, each collision sequence consists of at least six images. Out of the instantaneous data the mean droplet velocities are calculated and the droplet contact point is estimated by the velocities and the last droplet position (see Figure 3 right).
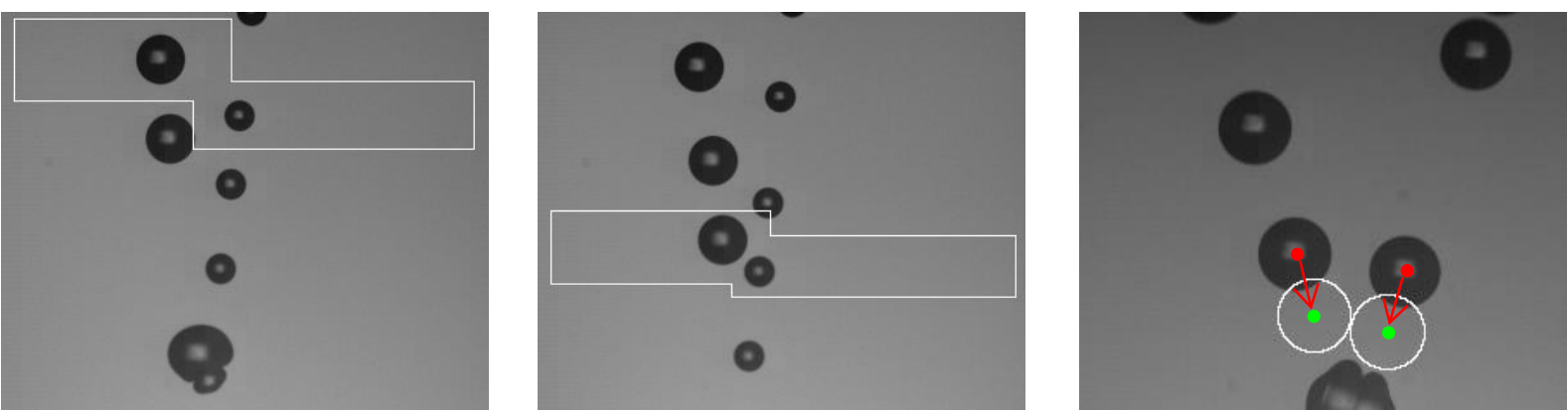

Figure 3. Variable box for droplet detection, left: first image of collision pair with large eccentricity, middle: last image before collision, right: estimated droplet contact point

The contact point position is used to calculate the non-dimensional impact parameter $\mathrm{B}$, which describes only the geometry of the collision without any liquid properties. The impact parameter is the sinus of the enclosed angle between the relative velocity and the position vector $\mathrm{P} 1,2$ of the droplets centre points (see Figure 4).

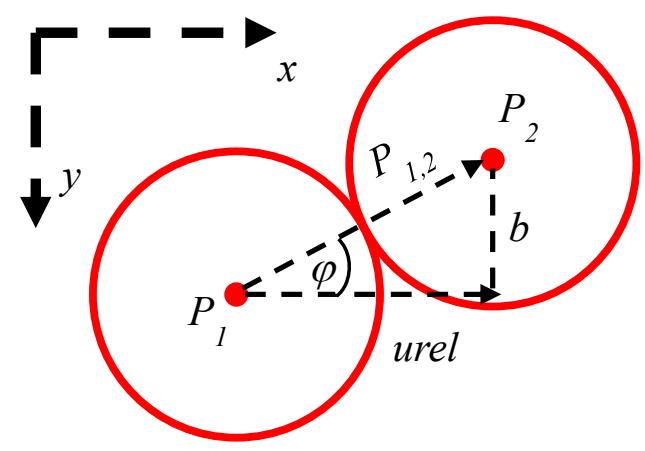

Figure 4. Droplet collision geometry [7]

The mean droplet relative velocity and diameter are used for the other non-dimensional numbers: Weber number We, size ratio (see Eq. 4) and the droplet Reynolds number Re (see Eq. 5). The effect of the viscosity is included in the Ohnesorge number Oh.

$$
\begin{aligned}
& B=\frac{2 b}{d_{1}+d_{2}}=\sin \varphi \\
& W e=\frac{\rho d_{1} u_{r e l}^{2}}{\sigma}
\end{aligned}
$$




$$
\begin{aligned}
& O h=\frac{\mu}{\sqrt{\rho \sigma d_{1}}} \\
& \Delta=\frac{d_{1}}{d_{2}} \\
& R e=\frac{\sqrt{W e}}{O h}=\frac{\rho d_{1} u_{r e l}}{\mu}
\end{aligned}
$$

For the collision outcome prediction the authors focus in this study only on the boundary line between stretching separation and coalescences. The Ashgriz and Poo [2] approach (see Eq. 6) for water droplets takes into consideration the size ratio, but not on the liquid properties. For higher viscos liquids the liquid properties had to be included. Based on this, Sommerfeld [5] improved the Gotaas et al. model (see Eq. 7) by implementing a dependency of Oh and the constant $C_{a}$ (see Eq. 8), and setting $C_{b}=1$.

$$
\begin{aligned}
& W e=\frac{4\left(1+\Delta^{3}\right)^{2}\left[3(1+\Delta)(1-B)\left(\Delta^{3} \phi_{S}-\phi_{l}\right)\right]^{0.5}}{\Delta^{2}\left[\left(1+\Delta^{3}\right)-\left(1-B^{2}\right)\left(\phi_{S}+\Delta^{3} \phi_{l}\right)\right]} \\
& B=\frac{C_{a}}{\sqrt{W e}}\left[1+C_{b} \frac{\mu}{\sigma}\left(\frac{\rho d_{1}}{\sigma}\right)^{0.5}\right] \\
& C_{a}=2.63-7.2 \cdot O h+7.86 \cdot O h^{2}+1.4 \cdot O h^{3}
\end{aligned}
$$

\section{Results and discussion}

The collision maps B over We are approved to describe the collision outcome of spray processes. The following collision maps are representing the complete outcome of 20 Ma\% PVP solution with constant material properties (see Table 1) and variation of droplet sizes and size ratios (see Table 2). The experiments were conducted up to We $=100$, with a special resolution of $\mathrm{We}<10$ and including the whole range of the impact parameter $\mathrm{B}<1$. Beside the experimental collision outcome, the fit of Ashgriz and Poo [2] and Sommerfeld [5] are plotted in the collision maps. The Gotaas et al. model is ignored because of the two variable constants $\mathrm{C}_{\mathrm{a}}$ and $\mathrm{C}_{\mathrm{b}}$.

For a better understanding of the collision outcome, droplets with $\Delta=1$ collided in Case 1 and 2 (see Table 2). In the collision maps the identification of triple point plays an imported role, because for higher We-number the stretching separation fraction increases. In Case 1 the triple point is located at We $\approx 26$ (see Fig. 5 left). By increasing the droplet size to $560 \mu \mathrm{m}$, in Case 2 the triple point is also located at We $\approx 26$ (see Fig. 5 right). The critical We- number (beginning of reflexive separation) occur out of head-on collision [2] with small B. The critical We-number in Case 2 is shifted from $W e \approx 46$ in Case 1 to $W e \approx 37$, as result of the higher kinetic energy and the lower influence of the surface tension. For We $<16$ bouncing is the only collision outcome in Case 2 , in contrast to coalescence detected from the beginning in Case 1 (min. We-number). In Case 1 and 2 (see Fig. 5) the curve by Eq. 7 and 8 match with the lower boundary of the stretching separation, while the Ashgriz and Poo model [2] underpredict the boundary in Case 1 and fit in Case 2.
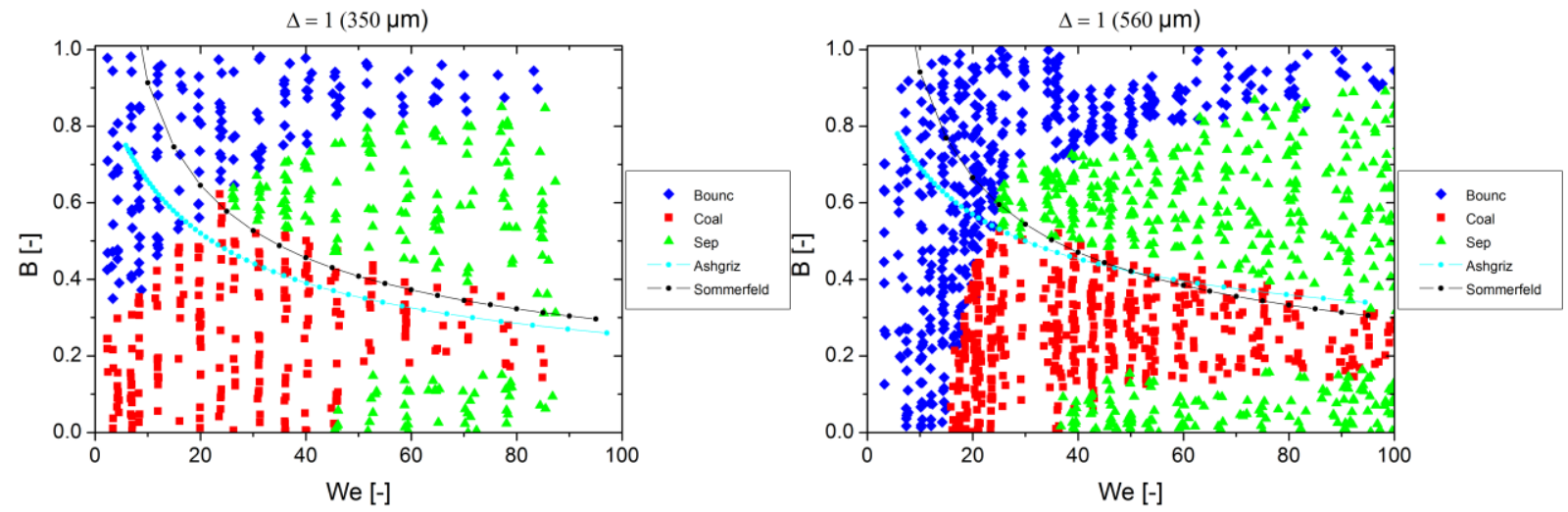

Figure 5. Collision maps of $5.5 \mathrm{mPas} \mathrm{PVP}$; left: Case $1, \Delta=1$, mean droplet size $=350 \mu \mathrm{m}$; right: Case $2, \Delta=1$, mean droplet size $=560 \mu \mathrm{m}$ 
According to the triple point results in Case 1 and 2, the point in Cases 3 to 5 (see Fig. 6) is located closed to We $\approx 26$. The critical We-number increases for smaller size ratio. A lack of data in Case 4 (see Fig. 6 top right), in the area in front of the reflexive separation and $B \approx 0.1$, suggests that the critical We-number is lower than We $\approx 52$. In opposite to the critical We-number the beginning of coalescences decreases for smaller size ratio. A lack of data in Case 3 (see Fig. 6 top left), in the area in front of the coalescence and $B \approx 0.45$, suggests that the beginning of coalescence is lower than $W e \approx 20$. In comparison to the Ashgriz and Poo model [2], which underpredict the lower boundary of the stretching separation, the Sommerfeld [5] curve (see Fig. 6 centre) shows a deviation from the experiments only in Case 5.

For small We-numbers (depending on the liquid properties) early coalescence could be observed. This outcome was detected for one collision in Case 3 (see Fig. 6 top left).
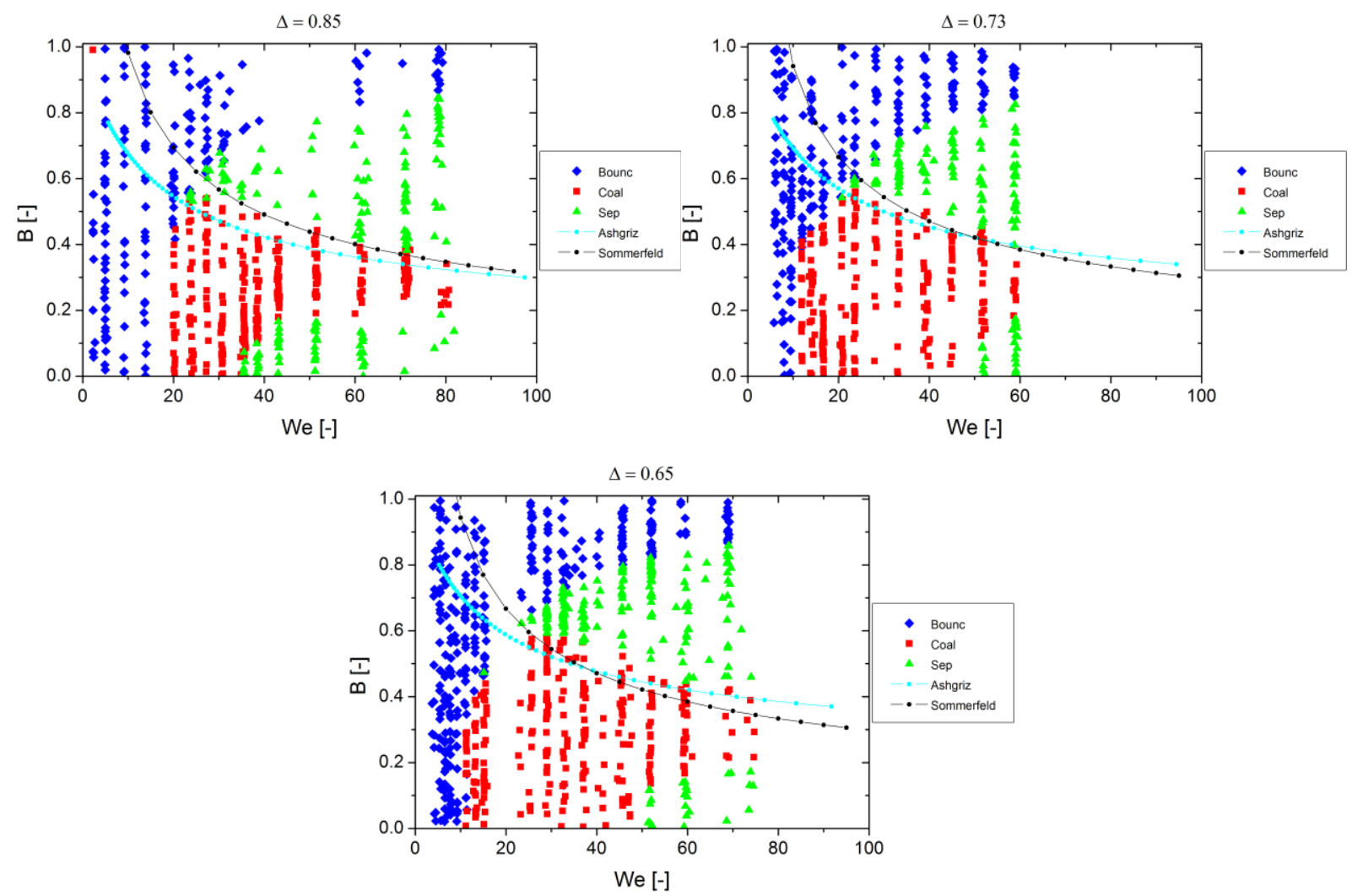

Figure 6. Collision maps of $5.5 \mathrm{mPas} \mathrm{PVP}$; top left: Case $3, \Delta=0.85$; top right: Case $4, \Delta=0.73$; centre: Case $5, \Delta=0.65$

The relevant We-numbers, related to the collision maps of Case 1 - 5 (see Fig. 5, 6), and the corresponding Ohnumbers are used to predict the dependency between Oh and We (see Fig. 7) [7].

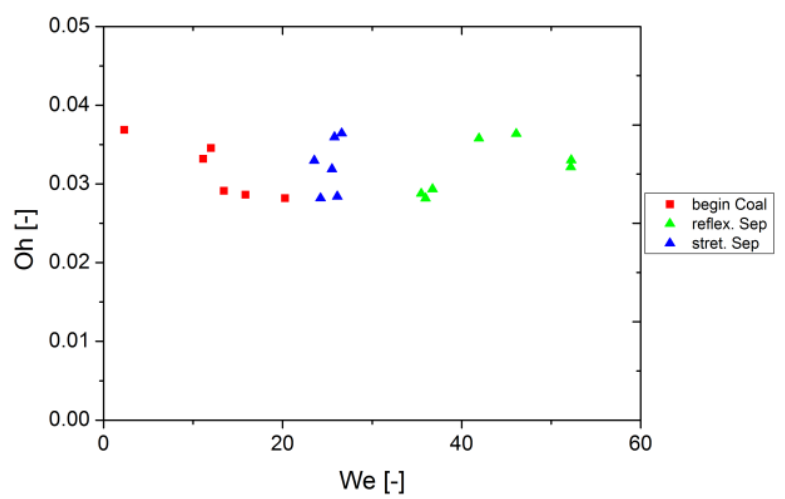

Figure 7. Onset of coalescence, reflexive and stretching separation; for Cases 1 - 5 and two additional experiments with PVP (see Table 1), Case 6: $\Delta=1$, mean droplet size $=360 \mu \mathrm{m}$; Case 7: $\Delta=1$, mean droplet size $=570 \mu \mathrm{m}$ 


\section{Conclusions}

The influence of droplet size properties was identified by the use of a new droplet detection method. For different droplet size properties of the same liquid the important We-numbers: beginning of coalescence, beginning stretching separation and beginning of reflexive separation were presented. The results for the coalescence and reflexive separation indicate a dependency between Oh-number and We-number. Smaller size ratio resulting in smaller We-number for the beginning of coalescence and larger We-number for the beginning of reflexive separation. The collision maps of the five Cases are showing that triple point is not influenced by the droplet size properties. The Sommerfeld [5] approach is demonstrating that the lower boundary of stretching separation can be described only by liquid properties and droplet size.

\section{Nomenclature}

b Lateral displacement of the droplet centres upon collision [m]

B Non-dimensional impact parameter [-]

$\mathrm{C}_{\mathrm{a}}, \mathrm{C}_{\mathrm{b}} \quad$ Parameter of Jiang et al. [3] model

$\mathrm{d}_{1} \quad$ Small droplet diameter [m]

$\mathrm{d}_{2} \quad$ Large droplet diameter [m]

Oh Ohnesorge number [-]

$\mathrm{Re} \quad$ Droplet Reynolds number [m]

urel Relative velocity

We Weber number

$\Delta \quad$ Size ratio [-]

$\mu \quad$ Dynamic viscosity of the fluid [Pa s]

$\Phi_{\mathrm{S}}, \Phi_{\mathrm{l}} \quad$ Parameter for small and large droplets in the Ashgriz and Poo [2] model [-]

$\rho \quad$ Density of the liquid $\left[\mathrm{kg} / \mathrm{m}^{3}\right]$

$\sigma \quad$ Surface tension of the liquid $[\mathrm{N} / \mathrm{m}]$

$\varphi \quad$ Enclosed angle between the relative velocity and the position vector $\mathrm{P} 1,2$ of the droplets centre points $\left[^{\circ}\right]$

\section{References}

[1] Estrade, J.-P., Carentz, H., Lavergne, G., Biscos, Y. "Experimental investigation of dynamic binary collision of ethanol droplets - a model for droplet coalescence and bouncing", 1999, International Journal of Heat and Fluid Flow 20, pp. 486-491.

[2] Ashgriz, N.; Poo, J.Y.:"Coalescence and separation in binary collisions of liquid drops", 1990, Journal of Fluid Mechanics 221, pp. 183-204.

[3] Jiang, Y. J., Umemura, A. and Law, C.K.: "An experimental investigation on the collision behavior of hydrocarbon droplets", 1992, Journal of Fluid Mechanics 234, pp. 171-190.

[4] Gotass , C., Havelka, P. Jakobson, H. A. and Svendsen H.F, Hase, M., Roth, N., and Weigand, B. : "Effect of viscosity on droplet droplet collision outcome Experimental study and numerical comparison", 2007, Physics of Fluids 19, 102106

[5] Sommerfeld, M., Sep. 4.-7. 2011, 27th European Conference on Liquid Atomization and Spray Systems.

[6] Kuschel, M., Sommerfeld, M., Investigation of droplet collisions for solutions with different solids content, 2013, Exp Fluids 54:1440.

[7] Sommerfeld, M, Kuschel, M., "Modelling droplet collision outcomes for different substances and viscosities", 2016, Exp Fluids 57:187. 